\title{
Avaliação do nível de conhecimento e aplicabilidade da Classificação Internacional de Funcionalidade, incapacidade e saúde (CIF) em profissionais de fisioterapia
}

\author{
Assessment of the level of knowledge and applicability of the International Classification of \\ Functionality, disability and health (CIF) in physiotherapy professionals \\ Evaluación del nivel de conocimientos y aplicabilidad de la Clasificación Internacional de \\ Funcionalidad, discapacidad y salud (CIF) en profesionales de fisioterapia
}

Recebido: 20/04/2021 | Revisado: 26/04/2021 | Aceito: 04/05/2021 | Publicado: 16/05/2021

\author{
Gerson de Melo da Silva \\ ORCID: https://orcid.org/0000-0002-5780-9770 \\ Faculdade Facottur, Brasil \\ E-mail: gmspyro2012@gmail.com \\ Lucas de Oliveira Santos \\ ORCID: https://orcid.org/0000-0002-9335-8727 \\ Faculdade Facottur, Brasil \\ E-mail: lucasoliveira1278@gmail.com \\ Augusto Cesar Bezerra Lopes \\ ORCID:https://orcid.org/0000-0002-3566-0055 \\ Faculdade Facottur, Brasil \\ E-mail: augustocesarb185@gmail.com \\ Edna Silva de Melo \\ ORCID: https://orcid.org/0000-0002-2791-1360 \\ Faculdade Facottur, Brasil \\ E-mail: edna.melo1@hotmail.com \\ Juliana Alves do Monte \\ ORCID: https://orcid.org/0000-0003-2968-5763 \\ Universidade Católica de Pernambuco, Brasil \\ E-mail: julianamontefisio@gmail.com \\ Marianna de Melo Salemi \\ ORCID: https://orcid.org/0000-0001-5804-6181 \\ Universidade Federal de Pernambuco, Brasil \\ E-mail: mariannasalemifisio@gmail.com \\ Érica Patrícia Borba Lira Uchôa \\ ORCID: https://orcid.org/0000-0003-4099-1876 \\ Universidade Católica de Pernambuco, Brasil \\ E-mail: ericaluchoa@gmail.com \\ Vanessa Maria da Silva Alves Gomes \\ ORCID: https://orcid.org/0000-0002-6866-6808 \\ Universidade Federal de Pernambuco, Brasil \\ E-mail: vanessaalvesfta@gmail.com
}

\begin{abstract}
Resumo
Objetivo: O presente estudo teve como objetivo avaliar o nível de conhecimento e aplicabilidade da CIF em profissionais de fisioterapia. Métodos: Trata-se de um estudo exploratório, descritivo de corte transversal com abordagem quantitativa, envolvendo fisioterapeutas que residem em Pernambuco e são ativos no Conselho Regional de Fisioterapia e Terapia Ocupacional da primeira região (CREFITO-1). Os voluntários responderam a um questionário sobre a CIF desenvolvido pelos pesquisadores através da plataforma Google Forms. Os dados coletados foram tabulados por meio de uma planilha, e desta foram apresentados descritivamente e, posteriormente, tendo seus resultados comparados através de testes considerando o nível de segurança <0,05 pelo software Statistical Package for the Social Sciences (SPSS), versão 22.0. Resultados: A amostra do presente estudo foi composta por 55 fisioterapeutas, com um predomínio do sexo feminino e profissionais da área de traumato ortopedia (34,4\%) e neurologia (23,3\%). Dentre os participantes, 94,5\% afirmaram conhecer a CIF, porém apenas $28,8 \%$ dos profissionais aplicam esta classificação em seu ambiente de trabalho, como uma ferramenta de avaliação e evolução do paciente durante as condutas. Conclusão: Conclui-se que, apesar dos fisioterapeutas conhecerem a CIF, muitos não a utilizam em sua pratica clínica e, dos participantes que utilizam, só a aplicam quando realmente se sentem preparados para sua utilização.
\end{abstract}

Palavras-chave: Classificação internacional de funcionalidade, Incapacidade e saúde; Fisioterapia; Saúde. 


\begin{abstract}
Aim: The present study aimed to assess the level of knowledge and applicability of the ICF in physiotherapy professionals. Methods: This is an exploratory, descriptive cross-sectional study with a quantitative approach, involving physiotherapists who live in Pernambuco and are active in CREFFITO-1. The volunteers answered a questionnaire about the CIF developed by the researchers through the Google Forms platform. The collected data were tabulated by means of a spreadsheet, and this was presented descriptively and, later, having their results compared through tests considering the security level $<0.05$ by the software Statistical Package for the Social Sciences (SPSS), version 22.0. Results: The sample of the present study consisted of 55 physical therapists, with a predominance of females and professionals in the field of trauma orthopedics (34.4\%) and neurology (23.3\%). Among the participants, $94.5 \%$ claimed to know the ICF, but only $28.8 \%$ of the professionals apply this classification in their work environment, as a tool for assessing and evolving the patient during conduct. Conclusion: It is concluded that, although the physiotherapists know the ICF, many do not use it in their clinical practice and, of the participants who use it, only apply it when they really feel prepared for its use.
\end{abstract}

Keywords: International classification of functioning; Disability and health; Physiotherapy; Health.

\title{
Resumen
}

Objetivo: El presente estudio tuvo como objetivo evaluar el nivel de conocimiento y aplicabilidad de la CIF en profesionales de la fisioterapia. Métodos: Se trata de un estudio exploratorio, descriptivo, transversal, con abordaje cuantitativo, que involucró a fisioterapeutas residentes en Pernambuco y activos en CREFFITO-1. Los voluntarios respondieron un cuestionario sobre el CIF desarrollado por los investigadores a través de la plataforma Google Forms. Los datos recolectados se tabularon mediante una hoja de cálculo, y esta se presentó de manera descriptiva y, posteriormente, se compararon sus resultados mediante pruebas considerando el nivel de seguridad $<0.05$ mediante el software Statistical Package for the Social Sciences (SPSS), versión 22.0. Resultados: La muestra del presente estudio estuvo conformada por 55 fisioterapeutas, con predominio de mujeres y profesionales en el campo de la ortopedia traumatológica $(34,4 \%)$ y neurología $(23,3 \%)$. Entre los participantes, el 94,5\% afirmó conocer la CIF, sin embargo solo el $28,8 \%$ de los profesionales aplica esta clasificación en su entorno laboral, como herramienta de valoración y evolución del paciente durante las conductas. Conclusión: Se concluye que, aunque los fisioterapeutas conocen el ICF, muchos no lo utilizan en su práctica clínica y, de los participantes que lo utilizan, solo lo aplican cuando se sienten realmente preparados para su uso.

Palabras clave: Clasificación internacional de funcionalidad, Discapacidad y salud; Fisioterapia; Salud.

\section{Introdução}

Em 2001 a 54 Assembleia da Organização Mundial de Saúde (OMS) publicou a Classificação Internacional de Funcionalidade, Incapacidade e Saúde (CIF), que integrou "A Família de Classificações Internacionais" da OMS, com o intuito de ampliar o entendimento da funcionalidade e da incapacidade. Em 2012 através da resolução $\mathrm{n}^{\circ} 452 / 2012$ do Conselho Nacional de Saúde (CNS), a CIF foi adotada para uso no Sistema Único de Saúde (SUS) (Araujo \& Neves, 2015; Belmonte, Chiaradia \& Belmonte, 2015; Regalado, Azevedo \& Pereira, 2015).

A CIF tem como objetivos principais: definir uma linguagem unificada e padronizada para descrição de saúde e a sua relação, facilitando a comunicação entre diferentes profissionais, entre eles os profissionais da saúde e possibilitar troca de informações entre países. O modelo da CIF fornece um sistema de codificação, no qual permite ao profissional avaliar e qualificar os prejuízos, limitações de realização e participação de uma atividade resultante de uma condição de saúde, além também de codificar os fatores externos que podem ser uma barreira ou facilitador para realização das atividades (Morettin 2012; Piexak, Cezar -Vaz \& Bonow, 2019).

$\mathrm{Na}$ área da saúde, a CIF é um mecanismo que pode ser utilizado por toda equipe multidisciplinar como fisioterapeutas, médicos, enfermeiros, entre outros. No entanto, o fisioterapeuta é o profissional que está mais relacionado com a CIF, devido a sua abordagem a cerca da funcionalidade, auxiliando os fisioterapeutas na avaliação e no diagnóstico cinético funcional do paciente, facilitando na escolha das melhores condutas para o tratamento fisioterapêutico (Castro, Pinto \& Almeida, 2015; Júnior et al., 2017).

Com a utilização da CIF, a fisioterapia está entrando em processo de reorientação tanto na fase primária quanto nas fases secundária e terciária da saúde, com um modelo individual terapêutico e especializado, utilizando de métodos técnicocientíficos sofisticados, com objetivo de trazer o paciente novamente às suas condições físicas e funcionais ideais, com foco 
em estratégias de tratamento e prevenção, voltadas ao desenvolvimento de políticas ligadas à funcionalidade humana e à educação em saúde, por tanto, faz-se necessária a promoção de abordagens integrais, enxergando o indivíduo tanto em seu aspecto clínico quanto social (Morettin, 2012; Ressler, 2017).

Atualmente existe um grande trabalho do sistema do Conselho Federal de Fisioterapia e Terapia Ocupacional (COFFITO) e do Conselho Regional de Fisioterapia e Terapia Ocupacional (CREFITO) em conjunto com instituições de ensino que formam profissionais de fisioterapia em mostrar a adequada utilização da CIF e difundir essa classificação entre profissionais e alunos, como preconizado pela Resolução no 370/2009 do COFFITO na formação acadêmica e profissional, recomendando a inclusão da CIF nas instituições formadoras de ensino superior, tanto na graduação, pós-graduação e extensão. No entanto, mesmo com a recomendação do COFFITO para que os fisioterapeutas utilizassem a CIF em suas vivências práticas desde 2009, muitos dos profissionais não aplicam CIF na sua rotina com os pacientes (Conselho Federal de Fisioterapia e Terapia Ocupacional, 2009; Ressler, 2017).

Considerando a necessidade e importância da implementação da CIF no que corresponde a fisioterapia, o presente trabalho visa avaliar o nível de conhecimento e aplicabilidade da CIF em profissionais de fisioterapia, avaliando os níveis de conhecimento e aplicabilidade desta pelos fisioterapeutas.

\section{Metodologia}

Trata-se de um estudo exploratório, descritivo de corte transversal com abordagem quantitativa. O estudo foi aprovado pelo Comitê de Ética e Pesquisa da Faculdade de Medicina de Olinda sob o CAAE: 36607520.3.0000.8033 e $n^{\circ}$ do parecer "4.334.986". O período do estudo foi realizado entre os meses de março a dezembro de 2020.

O recrutamento foi feito de forma intencional, através da divulgação da proposta por meio de Redes Sociais (Whatsapp, Instagram e Facebook). Além disso, houve o apoio do CREFITO-1, que realizou a divulgação da pesquisa, por meio de envio de e-mails para os profissionais que tinham cadastros ativos no conselho. O procedimento de coleta foi realizado através da utilização da ferramenta Google Forms, sendo esta amostra constituída por 55 profissionais de fisioterapia com vínculo ativo no CREFITO-1 e que residem em Pernambuco durante o período da coleta.

Os critérios de inclusão do presente estudo foram: profissionais de ambos os sexos e que atuam na área de fisioterapia, com critérios de exclusão sendo: profissionais que não residem e atuam no estado de Pernambuco e profissionais que não aceitaram o Termo de Consentimento Livre e Esclarecido (TCLE).

Após assinatura do TCLE, os participantes receberam um questionário on-line desenvolvido pelos pesquisadores da presente pesquisa na plataforma Google Forms constituído por 21 perguntas (dicotômicas e Likert), as quais 8 foram de caráter sociodemográfico e 13 questões relacionadas a CIF.

Os desfechos investigados durante a coleta de dados foram: o conhecimento sobre a CIF, aplicabilidade da CIF e motivos pela não utilização desta. Após os voluntários responderem completamente o questionário, os dados coletados foram tabulados por meio de uma planilha de dados elaborada no Microsoft Excel 2013, de onde foram apresentadas a estatística descritiva por meio de distribuição de frequências e percentagem. Posteriormente foi aplicado o Teste Qui-Quadrado de Pearson pelo Statistical Package For the Social Sciences (SPSS), versão 22.0, para comparar os resultados, considerando o nível de segurança $<0,05$.

\section{Resultados}

A amostra do presente estudo foi composta por 55 fisioterapeutas, dentre os participantes, o sexo feminino foi de maior prevalência (67,3\%). A área da fisioterapia que contou com o maior número de participantes foi a de traumato-ortopedia $(36,4 \%)$, seguido de neurologia (23,6\%). A maioria dos participantes $(80 \%)$ realizou a graduação em uma instituição privada 
(Tabela 1). Quanto ao tempo de graduação, foram prevalentes os fisioterapeutas com mais de oito anos de formados (29,1\%), seguido dos que tinham entre um a dois anos de formados (23,6\%). Sobre o nível de escolaridade há um predomínio maior $(58,2 \%)$ de participantes com pós-graduação (Tabela 1).

Tabela 1: Caracterização sóciodemográfica de todos os participantes da amostra.

\section{Variáveis}

\section{Sexo}

Feminino

$37(67,3 \%)$

Masculino

$18(32,7 \%)$

\section{Idade n(\%)}

20 a 25 anos

$15(27,3 \%)$

26 a 30 anos

$19(34,5 \%)$

31 a 35 anos

$7(12,5 \%)$

36 a 40 anos

$3(5,5 \%)$

$>40$ anos

$10(18,2 \%)$

Tempo de Graduação - n(\%)

$<1$ ano

$4(7,3 \%)$

De 1 a 2 anos

$13(23,6 \%)$

De 2 a 4 anos

$11(20 \%)$

De 4 a 8 anos

$11(20 \%)$

$>8$ anos

Tipo de Instituição de Ensino Superior onde se graduou - n(\%)

Público

$11(20 \%)$

Privado

Região da instituição de ensino superior - n(\%)

Nordeste

$52(94,5 \%)$

Sudeste

Nível de escolaridade - $\mathbf{n}(\%)$

Graduação

Especialização

Pós-graduação (latu sensu)

Mestrado

$12(21,8 \%)$

Doutorado

Área de Especialidade - n(\%)

Traumato-Ortopedia

Neurologia

Pediatria

Gerontologia

Acupuntura

Osteopatia

Quiropraxia 


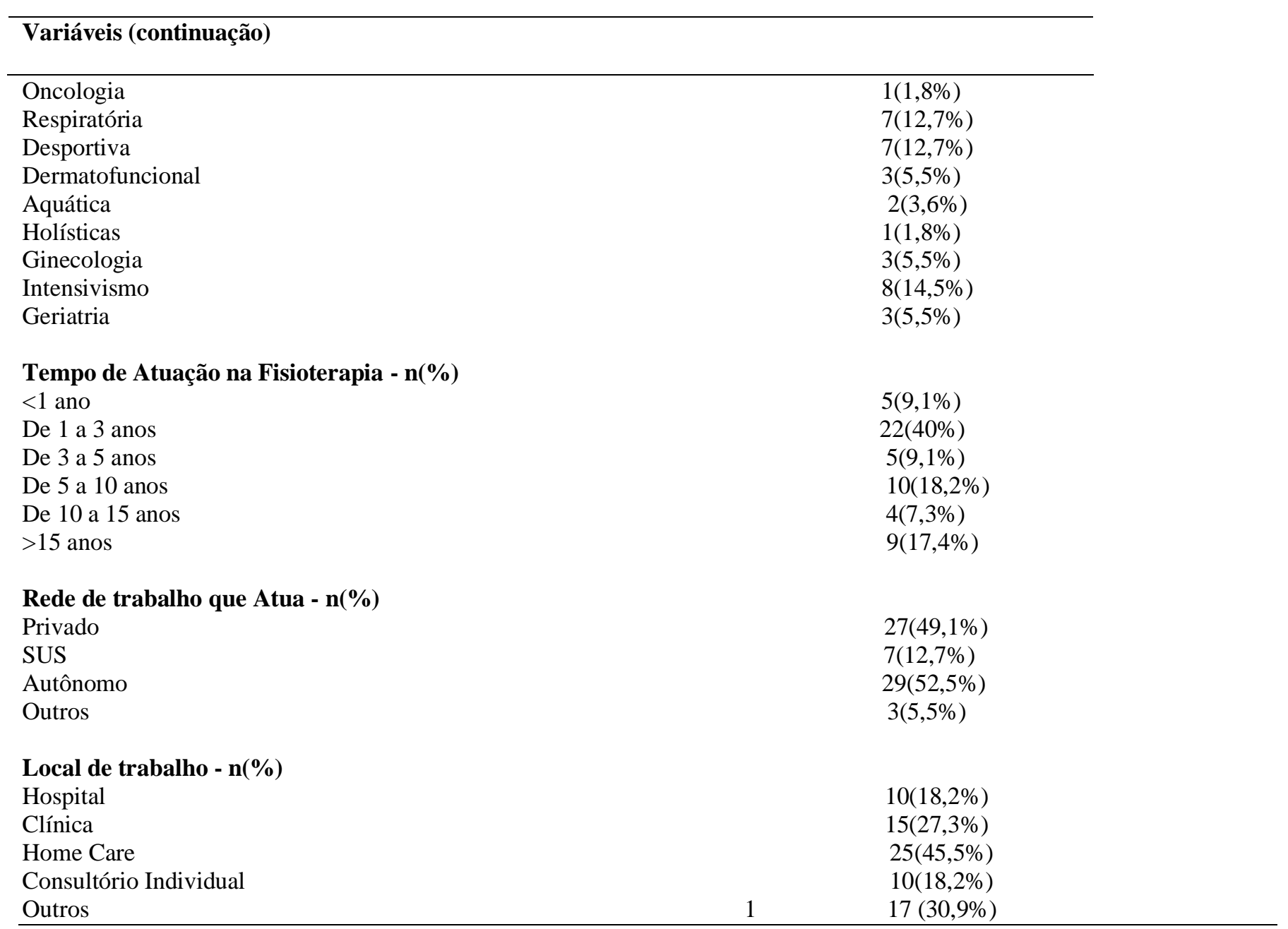

Fonte: Pesquisa direta (2020).

Com relação aos resultados do tópico conhecimento sobre a CIF, houve uma prevalência de participantes que relatou conhecê-la $(94,5 \%)$, cujo destes, 61,5\% relataram ter um nível regular de conhecimento. Com relação a utilização da CIF no ambiente de trabalho, dentre os profissionais que a conhecem, 37 (71,2\%) afirmaram não ter o conhecimento, enquanto 15 $(28,8 \%)$ relatam a utilizar. Daqueles que responderam não utilizar na prática clínica, 17 (45,9\%) profissionais não relataram os motivos, seguido de $16(43,2 \%)$ participantes que relataram ter conhecimentos insuficientes de sua utilização (Tabela 2).

Já para os profissionais que utilizam a CIF em seu ambiente de trabalho, 6 (40\%) relataram utilizá-la às vezes, tendo em $100 \%$ dos casos a finalidade de utilizar na avaliação. Por fim $10(66,7 \%)$ destes profissionais relataram estarem preparados para lançar mão da CIF em seu ambiente de trabalho (Tabela 3). 
Tabela 2: Conhecimento sobre a Classificação Internacional de Funcionalidade e fatores associados.

\section{Variáveis}

Você sabe o que é CIF? - n( \%)

Sim

Não

Qual seu nível de conhecimento sobre a CIF? - n( \%)

Muito Alto

Alto

Regular

Baixo

Muito Baixo

Por qual meio de informação você obteve conhecimento sobre a CIF pela primeira vez? - n( \%)

Livros/Artigos

Coffito/Crefito

$8(15,4 \%)$

Palestras/Cursos

$1(1,9 \%)$

Meio Acadêmico

$9(17,3 \%)$

Internet

$29(55,8 \%)$

Outros

$5(9,6 \%)$

$0(0 \%)$

Você teve conhecimento sobre a CIF na faculdade? - n( \%)

Sim

$32(61,5 \%)$

Não

$18(34,6 \%)$

Não sei dizer

Você utiliza a CIF em seu ambiente de trabalho? - n( \%)

Sim

Não

Se você não utiliza qual o possível motivo? - n( \%)

Falta de segurança

$0(0 \%)$

Conhecimento insuficiente de como utilizar

Dificuldade na aplicação da CIF

Não acredita que deve usar a CIF

Outros motivos

Fonte: Pesquisa direta (2020). CIF = Classificação Internacional de Funcionalidade.

Ao realizar a análise estatística utilizando o Teste Qui Quadrado de Pearson para cruzar as respostas de quem conhecia a CIF com as características da amostra, como tempo de graduação, tipo de instituição de ensino superior onde se graduou, nível de escolaridade, áreas de especialidade, tempo de atuação na fisioterapia, rede de trabalho que atua e local de trabalho, não foram apresentados diferença significativa ( $p>0,05)$. Também não houve diferença significativa ao cruzar as respostas da utilização/aplicabilidade da CIF com o nível de conhecimento, local de trabalho e área de especialidade ( $\mathrm{p}>0,05$ ). 
Tabela 3: Utilização da Classificação Internacional de Funcionalidade e fatores associados.

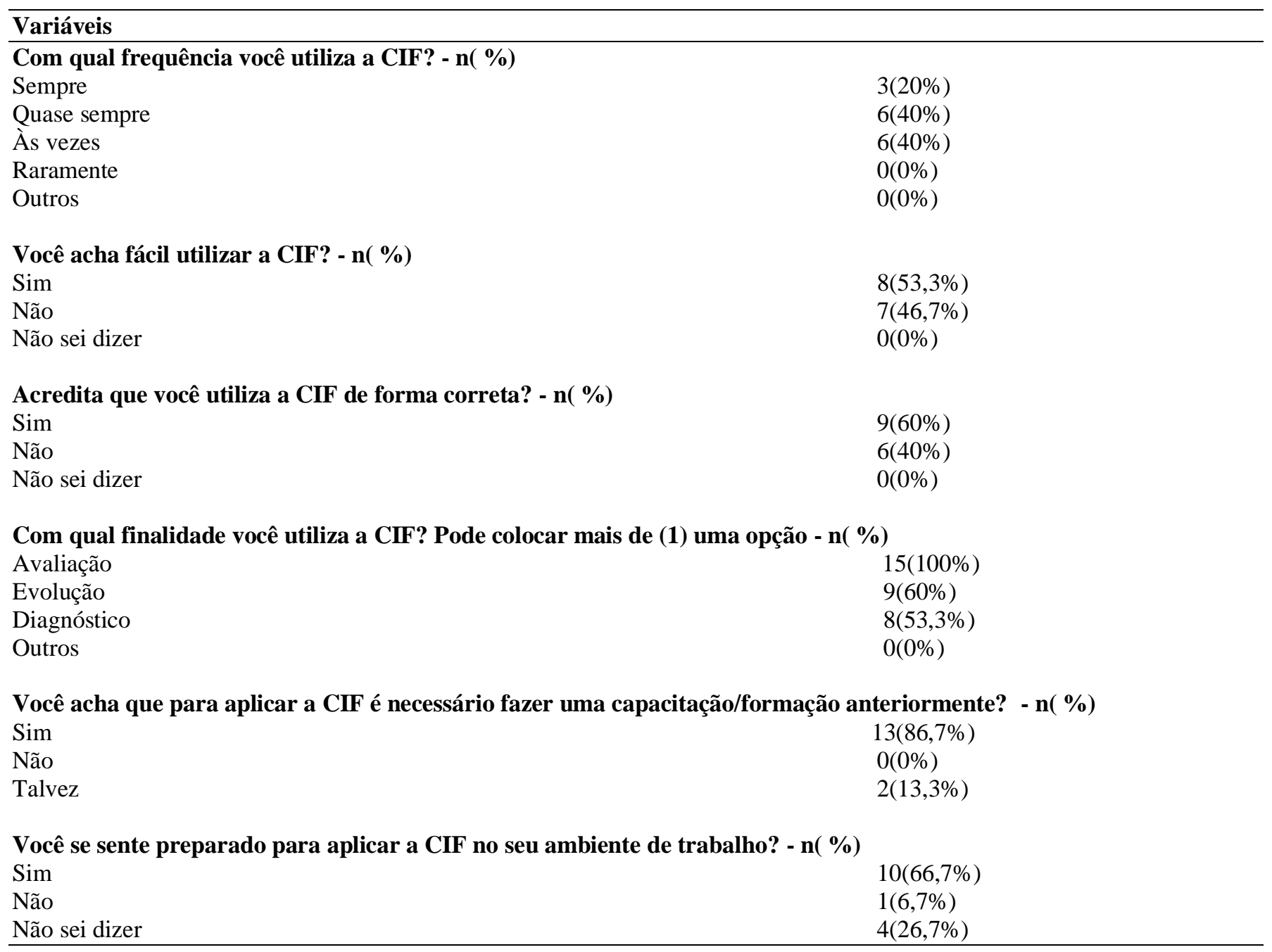

Fonte: Pesquisa direta (2020). CIF = Classificação Internacional de Funcionalidade.

\section{Discussão}

A CIF é uma ferramenta que pode ser utilizada por toda equipe multidisciplinar como fisioterapeutas, médicos e enfermeiros, contribuindo para uma abordagem multidimensional do paciente, utilizando uma linguagem universal entre os profissionais de saúde. (Castro, Pinto \& Almeida, 2015)

No presente estudo foi avaliado o nível de conhecimento da CIF pelos fisioterapeutas atuantes no estado de Pernambuco, sendo observado que a grande maioria (94,5\%) possui conhecimento regular desta, adquirido principalmente no meio acadêmico. Santos et al. (2020) em um estudo realizado com fisioterapeutas da cidade de Teresina - PI, também observou que grande parte dos profissionais (90,32\%) tinham conhecimento sobre a CIF e que o primeiro contato foi através da graduação. Com isso podemos destacar que estes achados sugerem que as instituições de ensino de graduação e especialização estão seguindo as recomendações do COFFITO/CREFITO que recomenda às instituições de ensino superior a trazer para os acadêmicos o conhecimento sobre a CIF.

Já Andrade et al. (2017), que avaliou o nível de conhecimento da CIF pelos profissionais de saúde, observou que a grande maioria dos participantes não conhecem a CIF (71\%) e, aqueles que conheciam a classificação, afirmaram ter um conhecimento precário. Uma possível justificativa para que os profissionais de saúde deste estudo tenham uma alta taxa de desconhecimento da CIF, diferente do estudo e dos achados de Santos et al. (2020), é que neste último a amostra é formada 
exclusivamente por fisioterapeutas, que são profissionais focados na abordagem cinético funcional, sendo assim, tendo uma maior possibilidade de conhecer esta classificação universal que está relacionada a funcionalidade do indivíduo.

Apesar de quase a totalidade da amostra afirmar conhecer a CIF (94,5\%), a maioria destes profissionais não a utilizam em seu ambiente de trabalho (71,2\%), sendo 45,9\% não relatou o motivo da não aplicação e seguidos de $43,2 \%$ destes que afirmaram não ter conhecimento suficiente para utilizá-la. Este resultado concorda com o estudo de Castro et al. (2015), que observou também que os fisioterapeutas da sua amostra não utilizavam a CIF na sua prática clínica, por afirmarem que é de grande complexidade. Estes dados demonstram a necessidade de fomento e oportunidades em cursos de capacitação, pois mesmo havendo informações sobre a CIF desde a graduação, é necessário preparar mais efetivamente sobre sua importância e a prática clínica da mesma através de informações e divulgação aos profissionais.

Ao analisar os participantes que utilizavam a CIF (28,8\%), observou-se que $40 \%$ destes relataram utilizar às vezes e outros $40 \%$ dos participantes relataram que quase sempre utilizam, concluindo-se que por mais que esses profissionais utilizem a CIF na sua prática clínica, a frequência do uso ainda é relativamente baixa. No entanto 53,3\% dos participantes consideram seu uso de fácil aplicação e acreditam que aplicam de forma correta.

Este resultado se contrapõe com o estudo de Santos et al. (2020), que teve como população estudada fisioterapeutas da área de neurofuncional, no qual 52\% relataram ter dificuldade para aplicar a CIF e com o de Belmonte et al. (2015) que contou como público alvo acadêmicos de fisioterapia do município de Florianópolis, e ao cruzar os dados, 46,9\% dos participantes afirmaram que sentem total dificuldade de utilizar a CIF e $34,6 \%$ afirmaram que concordaram parcialmente que tem dificuldade.

Uma diferença entre os estudos é que na presente pesquisa o questionamento sobre a dificuldade de utilização foi direcionado para aqueles fisioterapeutas que afirmaram utilizar a CIF na prática, já no estudo de Santos et al. (2020) e Belmonte et al. (2015) o questionamento foi para todos os participantes da pesquisa sem distinção se eles a aplicavam ou não. Outro ponto importante que pode justificar essas discordâncias nos achados é que em nosso estudo teve-se a importância de ampliar a amostra para todas as áreas de atuação, já no estudo de Santos et al. (2020) e Belmonte et al. (2015), realizaram a pesquisa apenas com fisioterapeutas atuantes em neurofuncional e com acadêmicos de fisioterapia respectivamente, restringindo a amostra para uma determinada população.

Uma das maiores dificuldades entre os profissionais de saúde é a linguagem padronizada para relatar a abordagem do paciente, documentando prontuários de forma restrita ao entendimento apenas pelos profissionais da mesma área de atuação ou profissão. Em nosso estudo, dentre os profissionais que utilizavam a CIF, 100\% a utilizavam para avaliação, seguido de $60 \%$ que também a utilizavam para evolução, estes dados corroboram com o estudo de Silva et al. (2015), que realizou um estudo observacional longitudinal descritivo, na UTI Cardiotorácica adulta, onde observou-se através das fichas de avaliação que os profissionais utilizam a CIF para avaliação e evolução dos pacientes no setor. A CIF tem um papel importante por promover uma linguagem padronizada, envolvendo questões biológicas, psicológicas, sociais e ambientais, possibilitando uma maior interação multiprofissional e universal.

Não houve diferenças estatísticas entre os participantes quanto ao nível de conhecimento e aplicabilidade com demais características do estudo. Uma possível justificativa deste resultado pode ser a adesão desta classificação que ainda está em fase de crescimento devido ser relativamente recente. Através do presente estudo observa-se que os fisioterapeutas tem conhecimento sobre a CIF, mas que a aplicação desta na prática clínica está em fase de progressão, mas quando comparado a estudos anteriores mencionados na literatura, observa-se resultados mais favoráveis para o uso da CIF.

Uma limitação deste estudo foi a pequena adesão dos profissionais, resultando em uma amostra pequena, mesmo tendo sido divulgado para uma grande parte dos fisioterapeutas, com a ajuda do CREFFITO-1. Além disso, o estudo limitou-se a fisioterapeutas atuantes no estado de Pernambuco. 


\section{Considerações Finais}

Conclui-se que, em relação ao conhecimento e aplicabilidade da CIF por profissionais de fisioterapia atuantes no estado de Pernambuco, a grande maioria dos participantes apesar de conhecer a CIF, sua a utilização na prática clínica está em fase de adequação e progressão. Dos que aplicam a CIF, estes a utilizam de forma precária em sua prática clínica.

Uma perspectiva futura é a importante ampliação deste estudo para todo o território brasileiro, elaborando estudos com maiores amostras, uma vez que há poucas publicações sobre a temática na literatura científica.

\section{Referências}

Andrade, L. E. L., et al. (2017). Avaliação do nível de conhecimento e aplicabilidade da Classificação Internacional de Funcionalidade, Incapacidade e Saúde. Rio de Janeiro: Saúde Debate.41(114), 812-823.

Araújo, E. S., \& Neves, S. F, P. (2015). Classificação Internacional de Funcionalidade, Incapacidade e Saúde, e-sus e tabwin: as experiências de Barueri e Santo André, São Paulo. Bahia: Revista Baiana de Saúde Pública, 39(2), 470-477.

Belmonte, L. M., Chiaradia, L. C. N., \& Belmonte, L. A. O. (2015). CIF nos cursos de graduação de fisioterapia da grande Florianópolis. Florianopolis: Revista Cientifica CIF Brasil, 2(2).

Castro, C. C., Pinto, C. N., \& Almeida, M. A. (2015). Conhecimento e aplicação da Classificação Internacional de Funcionalidade, Incapacidade e Saúde por fisioterapeutas de Fortaleza. Fortaleza: Revista Fisioterapia Saúde \&Funcional, 4(2), 6-13.

Conselho Federal de Fisioterapia e Terapia Ocupacional - COFFITO. (2009). Resolução 370 de 6 de novembro de 2009. Dispõe sobre a adoção da Classificação Internacional de Funcionalidade, Incapacidade e Saúde (CIF) da Organização Mundial de Saúde por Fisioterapeutas e Terapeutas Ocupacionais. Diário Oficial da União, n. 225, seção 1, página 101. <http://pesquisa.in.gov.br/imprensa/jsp/visualiza/index.jsp?data=25/11/2009\&jornal=1\&pagina=101>.

Junior, B. H. P., et al. (2017). Desenvolvimento de um software para suporte à avaliação fisioterápica baseado na Classificação Internacional de Funcionalidade, Incapacidade e Saúde. João Pessoa: Revista Eletrônica de Comunicação, Informação e Inovação em Saúde, 11(4).

Morettin, M. (2012). Classificação Internacional de Funcionalidade, Incapacidade e Saúde, versão crianças e jovens: elaboração de checklist para avaliação da funcionalidade em usuários de Implante Coclear. Tese (Doutorado em Ciências). Faculdade de Saúde Pública da Universidade de São Paulo.

Piexak D. R., Cezar-Vaz M. R, \& Bonow C. A. (2019). Classificação Internacional de Funcionalidade, Incapacidade e Saúde: uma análise de conteúdo. Revista Online de Pesquisa: Cuidado é Fundamental, 11(2), 363-369.

Regalado, C. R. I., Azevedo, I. G., Pereira, S. A. (2018). Classificação Internacional de Funcionalidade, Incapacidade e Saúde (CIF): ampliando o olhar sobre a criança com deficiência motora. Revista Movimenta. 11(3), 387-392.

Ressler, S. O uso prático da Classificação Internacional de Funcionalidade, Incapacidade e Saúde em fisioterapia. (2017). Dissertação (Mestrado em Saúde Coletiva). Universidade do Extremo Sul Catarinense.

Santos, L. N. L. (2020). Conhecimento e Utilização da CIF por Docentes Fisioterapeutas na Cidade de Teresina - PI. Revista Neurociencias. 28 , 1-14.

Silva. B. N. O., et al. (2016). Aplicação da classificação internacional de funcionalidade, incapacidade e saúde em Unidade de Terapia Intensiva Cardiotorácica. Fisioterapia Brasil. 17(2). 